\title{
ACTITUDES HACIA EL ABUSO DE PERSONAS DE MAYOR EDAD: ESTUDIO COMPARATIVO ENTRE CUBA Y PUERTO RICO
}

\author{
Carmen Delia Sánchez Salgado*
}

\section{Introducción}

$\mathrm{E}$ ste trabajo versará sobre los resultados de una investigación sobre la percepción y actitudes hacia el abuso y maltrato de personas de edad avanzada de un grupo de ancianos y ancianas mayores de 60 años en Puerto Rico y Cuba. La investigación forma parte de una más amplia multicultural y multiétnica realizada con personas mayores de 60 años de ambos sexos. La misma se inició en 1997 por iniciativa y auspicio del National Center on Elder Abuse con sede en Washington, D.C., Estados Unidos con la colaboración de los siguientes investigadores: Toshio Tatara (Director, National Center on Elder Abuse -Japón); Donna Benton (University of Southern California-Los Angeles); Melissa Talamantes (University of Texas Health Science Center, San Antonio, Texas); Yolanda Sánchez (University of Nevada, Reno Nevada); Ailee Moon (University of California, Los Angeles); Susan Tomita (University of Washington); Arnold Brown (Northern Arizona University, Arizona) y Carmen Delia Sánchez (Universidad de Puerto Rico). Los propósitos del estudio eran: (1) Descubrir cómo las personas ancianas

\footnotetext{
* Catedrática, Escuela Graduada de Trabajo Social, Universidad de Puerto Rico.
} 
definían el abuso y maltrato y cómo se sentían hacia las acciones que otros definían como abuso y maltrato hacia ellos; (2) A quiénes veían como responsables del abuso; (3) Cuán serio pensaban que era; y, (4) Qué pensaban debía hacerse al respecto. Participaron grupos de ancianos y ancianas áfrico-americanos, hispano- americanos, mexico-americanos, anglosajones blancos, japoneses, japoneses-americanos, coreanos, coreanos-americanos, indios americanos (Navajo), puertorriqueños y finlandeses. Posteriormente (1998) esta autora replicó la investigación con una muestra de personas ancianas en Matanzas, Cuba. Para fines de este trabajo solo se hará referencia a los hallazgos de la investigación de los participantes puertorriqueños y cubanos.

\section{Revisión de literatura}

En Puerto Rico y Cuba al igual que en muchos otros países el sector poblacional de sesenta años o más ha ido en aumento a un paso más rápido que el resto de la población. Este incremento reflejado en Puerto Rico desde los años 50, ha sido el resultado de una reducción en las tasas de nacimiento, la emigración de adultos jóvenes (edades entre 20 y 34) hacia los Estados Unidos y el regreso de emigrantes puertorriqueños de edad avanzada hacia la isla. La población de aquellos de sesenta o más años de edad aumentó de un $6 \%$ del total de la población en 1960 , a un $13.2 \%(465,736)$ en 1990 (Bureau of the Census, 1991). Las proyecciones para el año 2000 indican que el porcentaje del sector poblacional de 60 años o más aumentará a un $14.4 \%$ del total de la población. Los factores contribuyentes a tales proyecciones pueden incluir: el regreso de puertorriqueños de mayor edad hacia la isla, el envejecimiento de la generación "post-guerra" (baby-boom), la continua emigración de poblaciones jóvenes, la proporción de crímenes que tienden a impactar más a grupos de edad joven que a los grupos de mayor edad, la disminución en tasas de nacimientos y el aumento en el acceso a tratamientos médicos más avanzados y especializados.

El incremento potencial y actual en la proporción de población anciana, incluyendo el aspecto multigeneracional, presenta un reto a la provisión de servicios sociales y de salud a medida que aumenta el número de ancianos dependientes y frágiles. Claramente, estos cambios demográficos tienen implicaciones en el fenómeno y tragedia del abuso y maltrato a las personas ancianas. 
Más específicamente, si la población anciana está en aumento, de igual forma aumenta la posibilidad de maltrato (abuso o negligencia) a este sector poblacional. Sin ser optimista, esta correlación requiere de recursos comunitarios más extensos con los cuales manejar el maltrato a personas ancianas como un problema social que va en aumento. El cuidado comunitario implica proveer unos servicios sociales y de salud que permitan a la población anciana vivir tan independientemente como sea posible, en sus comunidades a pesar de los retos físicos y emocionales. Sin este cuidado intencional se puede esperar que sean más frecuentes los incidentes de abuso a personas ancianas.

En Puerto Rico, la epidemiología social del maltrato a las personas ancianas no está claramente delineada debido a la falta de notificaciones, la falta de data prevaleciente y carencia de estudios y recursos necesarios para desarrollar una base segura que describa el problema, cuantifique los datos e implemente remedios programáticos. El no reconocer que el maltrato a las personas ancianas sí ocurre y es, de seguro un problema social, es considerablemente contradictorio dado el notable y consistente aumento poblacional del sector de personas mayores de 60 años en las últimas décadas.

Las personas ancianas como sector poblacional, no han sido un foco de atención legislativo en cuanto a reconocimiento, definición, intervención y penalización del maltrato. Quizás, porque en Puerto Rico, el concepto de abuso o negligencia a personas ancianas, separado del maltrato familiar, no ha sido visualizado como un problema social en sí. A pesar de que el tema de la violencia familiar ha sido estudiado con bastante frecuencia, la violencia hacia los ancianos o ancianas por parte de miembros de su familia ha sido virtualmente ignorado hasta años recientes, en que se ha reconocido que las mujeres ancianas experimentan violencia doméstica en manos de sus esposos o de sus hijos o hijas.

Existe poco consenso entre los proveedores de servicio de que si el abuso o maltrato por los hijos e hijas cae bajo la clasificación de violencia doméstica. Uno de los pocos estudios sobre abuso de parte de los hijos hacia la mujer anciana, sugiere que estos casos comparten muchas similaridades con casos de abuso por parejas íntimas (Anetzberger, 1987). El estudio, que analizó 40 casos de abuso físico de parte de sus hijos a mujeres ancianas, caracterizó al maltratante como soltero, hombres de edad mediana con 
características patológicas, que vivían con sus madres. Los maltratantes proveían cuidado considerable a la madre anciana que probablemente estaba física o mentalmente incapacitada. Los maltratantes caían en una de tres categorías: hostiles, autoritarios o dependientes. Entre los factores que establecieron las condiciones para la violencia estaba "una intimidad prolongada y profunda" entre madre e hijo.

Los datos provistos por el Departamento de la Familia del Estado Libre Asociado de Puerto Rico en 1980, indican un total de 342 informes de abuso a personas mayores de 60 años. En 1990, los casos reportados aumentaron a 2,828. Estas estadísticas aumentaron nuevamente en 1996 a un total de 5,613 informes de abuso a personas mayores de 60 años. El servicio de Protección de esta agencia atendió en 1998 un total de 6, 076 casos. La Oficina del Gobernador para los Asuntos de la Vejez recibió 1,226 querellas de abuso en 1993 y 1,768 querellas en 1995 a través de sus diferentes oficinas de área. De acuerdo a estadísticas policíacas sobre violencia doméstica, al final del año 1994 se reportaron un total de 18,079 casos de violencia doméstica a esa agencia, de los cuales 115 fueron de mujeres mayores de 60 años (Babá et.al., 1996). La escasez de evidencia de abuso y maltrato a personas ancianas puede deberse en parte a que el maltrato es comúnmente perpetrado dentro del círculo familiar y no es notificado.

Los estudios revisados sobre el tema de abuso y maltrato a ancianos y ancianas en Puerto Rico, sugieren que muchos ancianos y ancianas en la isla están en riesgo o han sido maltratados por los proveedores de su cuidado, sean estos parientes o no-parientes (Muñoz, 1985; Arroyo et.al., 1992; Ramos Tossas, 1990; Babá et.al., 1996). La víctima típica de abuso y maltrato es una mujer mayor de 75 años, físicamente dependiente (Muñoz, 1985). El abusador usualmente es un hijo o hija adulto (Muñoz, 1985; Arroyo et.al., 1992).

En el estudio de Muñoz (1985), el descuido o negligencia constituyó la forma más común de abuso. Un cincuenta y tres porciento del abuso era llevado a cabo por una hija o un hijo y un $10 \%$ era llevado a cabo por el esposo o esposa. En un $40 \%$ de las situaciones la víctima había sido abusada por otro pariente.

Muñoz encontró que un $70 \%$ de los parientes o proveedores del cuidado estaban afectados por algún factor de tensión familiar. Entre los factores principales de tensión identificados estaban: la inhabilidad mental o física de un miembro de la familia (70\%); alcoholismo (31\%); 
condiciones de vivienda inadecuadas (30\%); falta de recursos económicos (18\%); desempleo (15\%) y enfermedades crónicas (10\%).

El estudio de Babá, Colón y Cruz (1996) se focalizó en las actitudes y conocimiento acerca de la violencia doméstica en mujeres de 60 años o más. Las investigadoras entrevistaron 100 mujeres ancianas residentes en las áreas urbanas de Puerto Rico. Las variables incluían aspectos sociodemográficos, la visión del rol femenino, actitudes hacia la violencia doméstica y el conocimiento de los aspectos legales y servicios para víctimas de violencia doméstica. Las entrevistadas identificaron la tolerancia (66\%) como un adjetivo descriptivo de la mujer y la agresividad (43\%) describiendo al hombre. En relación a las actitudes hacia la violencia doméstica, aunque la mayoría de las mujeres (99\%) repudiaban la violencia en una relación, un $30 \%$ consideró que el hombre puede ser violento cuando la mujer le es infiel. El 50\% argumentó que "una mujer debe preservar al padre de sus hijos no importa lo que pase"(p.131). No obstante, un $80 \%$ de las mujeres conocía la existencia de la Ley Contra la Violencia Doméstica, solo un $30 \%$ tenía algún conocimiento acerca de su contenido o los derechos de la persona victimizada.

A pesar de los cambios por los que ha pasado la familia puertorriqueña en las últimas décadas, aún retiene muchos aspectos de su identidad étnica hispánica y sus valores culturales. Uno de estos valores conocido como familismo, coloca un gran énfasis en la unidad familiar, apoyo a la integridad familiar y da forma y dirección al comportamiento de sus miembros. Al ser centrados en la familia, los miembros sienten obligación hacia sus parientes y una responsabilidad de servir en tiempos de crisis. De igual manera, la lealtad hacia la familia abstiene a los miembros ancianos de hablar sobre el maltrato. Existe evidencia que sugiere que algunas víctimas de abuso y maltrato se niegan a tomar acción legal contra los perpetradores por valores familiares que les mueven a proteger al maltratante de castigo.

Por otro lado, los valores familiares pueden interferir en la visibilidad del abuso y negligencia de la población anciana. Las familias no toleran la intromisión de extraños en sus asuntos. Tanto los ancianos maltratados como los maltratantes se adhieren a una conspiración de silencio y rechazan cualquier intervención profesional cuando se detecta abuso. Estos valores son más fuertes entre las personas mayores en la familia. Variables tales como lazos familiares, lealtad, relaciones de interdependencia hacen más difícil el que la víctima vaya en busca de ayuda externa. 
Las definiciones de abuso y maltrato se hacen por estatutos de ley o dentro de estudios en particular. Al igual que otras manifestaciones de violencia doméstica, el abuso y maltrato de ancianos no está confinado a un grupo étnico o cultural (Pillemer y Finkelhor, 1988, Quinn y Tomita, 1997). Sin embargo, solo algunos estudios pequeños han dirigido su atención a interpretaciones multiculturales del abuso y maltrato de personas ancianas (Griffin, 1994, Stein, 1991, Moon y Williams, 1993). Lo que estos estudios sugieren es que existen diferencias culturales entre grupos étnicos en cuanto a la percepción y definición del abuso y maltrato. El estudio de Moon y Williams (1993) dirigió su atención a cómo un grupo de 30 ancianos afro-americanos, caucasioamericanos y coreano-americanos $(\mathrm{N}=90)$ cualificaban ciertos escenarios para la presencia de abuso y maltrato, severidad del abuso y aspectos más abusivos de la situación. Los resultados sugirieron que un porcentaje mayor de afro-americanos cualificaron los escenarios abusivos en comparación con los otros dos grupos. Los coreanoamericanos fueron los que menos visualizaron los escenarios como abusivos. Los hallazgos de los pocos estudios en esta área dan énfasis a la necesidad de entender el abuso y maltrato dentro de los contextos culturales. Más aún, no sólo deben entenderse las percepciones de abuso y maltrato que se tiene en diversos grupos culturales y étnicos, sino desde la perspectiva de la misma población anciana.

La mayoría de los estudios sobre abuso y maltrato de ancianos o ancianas han abordado el tema desde la perspectiva de investigadores y practicantes. Específicamente, las definiciones y clasificaciones del abuso y maltrato utilizadas en diversas investigaciones se han desarrollado con poco o ningún insumo de la población de edad mayor. A pesar de que varios investigadores e investigadoras han buscado desarrollar una definición universal o "standard' del abuso o maltrato de ancianos, se ha omitido a la persona anciana de este proceso. Como resultado de esto puede surgir conflicto entre las personas ancianas, los practicantes y los investigadores en cuanto a si una situación envuelve abuso o maltrato y qué intervención será la más adecuada. Las personas ancianas pueden percibir o juzgar la situación de maltrato de manera diferente (Moon, A. y Williams, O.J., 1993). No existe mucha investigación en torno a cómo la persona anciana percibe e identifica un caso de maltrato y si las percepciones son consistentes con las clasificaciones de tal comportamiento por parte de profesionales. Estas inquietudes movieron a los investigadores e investigadoras a llevar a cabo este estudio. 


\section{Metodología}

El objetivo del estudio era obtener información respecto a como las personas ancianas percibían el abuso y maltrato hacia ellas; sus actitudes hacia notificarlo a las autoridades y cómo estas percepciones y actitudes pueden diferir de una cultura a otra.

Como se señalara previamente, este estudio forma parte de uno más amplio en que participaron diversos grupos étnicos incluyendo tres grupos de habla hispana. El instrumento fue adaptado de uno utilizado por la Dra. Ailee Moon (1993) en un estudio previo, que incluía una escala de 11 aseveraciones para medir actitudes sobre el abuso y maltrato. El cuestionario utilizado fue traducido a diversos idiomas y la versión en español fue revisado por tres profesionales en Estados Unidos y Puerto Rico para asegurarse que las palabras y frases eran congruentes con el uso del español en los diversos grupos. Para fines de esta presentación solo se analizaron las muestras de los puertorriqueños y los cubanos. Los datos se obtuvieron mediante entrevistas estructuradas cara a cara con dos grupos de 100 hombres y mujeres mayores de 60 años en Cuba y Puerto Rico. Las entrevistas se llevaron a cabo en sus hogares, centros de actividades múltiples o lugares de trabajo.

\section{Hallazgos principales}

\section{Puertorriqueños}

Las personas ancianas puertorriqueñas participantes del estudio estaban entre las edades de 62 a 92 años, siendo la mediana de edad 75 años. La gran mayoría eran mujeres (81\%) y sólo 19 eran hombres. Todas las personas, excepto una, nacieron y se criaron en Puerto Rico. Casi la mitad (49\%) eran viudas. Un $20 \%$ estaban casados, $22 \%$ divorciados o separados y $9 \%$ nunca se había casado. El promedio de hijos vivos era 3, y fluctuaba entre 12 y uno. Una cuarta parte de las personas entrevistadas no había tenido hijos o hijas. La educación formal promedio fue de 8 años de escolaridad y un $31 \%$ tenía menos de seis años de escolaridad. El $63 \%$ vivían solos. Entre las personas que vivían acompañadas, $16 \%$ vivía con su cónyuge, $11 \%$ con hijos o hijas solteras o casadas y el resto con otros familiares entre los que se pueden mencionar nietos o nietas, hermanos o hermanas u otros familiares. La mayoría de las personas entrevistadas $(39 \%)$ consideraba su estado de salud buena, $37 \%$ regular, $18 \%$ 
excelente y $5 \%$ pobre. Cerca de tres cuartas partes $(70 \%)$ estaba totalmente retirada, $12 \%$ estaban trabajando parcial o totalmente y $13 \%$ nunca habían trabajado fuera del hogar para empleos remunerados. La mayoría de los participantes vivían en residencia de su propiedad (61\%), en casa alquilada un $24 \%$, en apartamentos subsidiado $11 \%$ y 4 personas vivían en casas de otras personas.

\section{Cubanos}

Las personas ancianas cubanas que participaron en el estudio estaban entre las edades de 60 a 92 años, siendo la mediana de edad 68 años. La mayoría eran hombres (54\%) y el $46 \%$ eran mujeres. Todas las personas nacieron y se criaron en Cuba. Sobre una cuarta parte $(26 \%)$ de las personas eran viudas. La mayoría estaban casadas (39\%). El $21 \%$ eran divorciados o separados y un $14 \%$ nunca se había casado. El número de hijos o hijas vivos fluctuaba entre dos y seis siendo el promedio de 2 , aunque un $23 \%$ de las personas entrevistadas no había tenido descendientes. La educación formal promedio fue de 6 años y un 34\% tenía menos de seis años de escolaridad. En contraste con la muestra puertorriqueña, sólo un $30 \%$ de la muestra cubana vivían solos. Entre las personas que vivían acompañadas, $34 \%$ vivía con su cónyuge, $31 \%$ con hijos o hijas solteras o casadas y el resto con otros familiares entre los que se pueden mencionar nietos o nietas $(16 \%)$ hermanos o hermanas $(6 \%)$ $\mathrm{u}$ otros familiares (10\%). La mayoría de las personas entrevistadas (49\%) consideraba su estado de salud buena, $48 \%$ regular y sólo un $3 \%$ pobre. La gran mayoría $(80 \%)$ estaba totalmente retirada, 3\% parcialmente retiradas y un $17 \%$ nunca habían trabajado fuera del hogar o en empleos remunerados. La mayoría de los participantes vivían en residencia de su propiedad (77\%), en casa alquilada un $6 \%$ y $17 \%$ vivían en casas de otras personas.

Para fines de conocer las actitudes y percepciones sobre el abuso y maltrato y su informe, se les ofreció a las personas entrevistadas unas aseveraciones que en la literatura describían el abuso y maltrato hacia las personas ancianas. Las respuestas se clasificaron en: Abuso psicológico, Abuso físico, Abuso financiero, Negligencia, Dilemas éticos de auto-determinación, Percepción sobre causas y magnitud del abuso o maltrato, y Actitudes hacia notificar el abuso o maltrato. Las siguientes tablas muestran los hallazgos en estas categorías para ambos grupos. 


\section{A: Puertorriqueños:}

Aseveración

\section{Abuso Psicológico}

Esta bien que un

hijo adulto le grite ocasionalmente a sus padres o madres ancianas.
N De

Desacuerdo

Depende Acuerdo

No Sé

Ser ignorado, excluido $o$ aislado por un miembro de la familia es tan dañino como ser abusado físicamente.

$6 \%$

$1 \%$

$1 \%$

\section{Abuso Físico}

Cuando un hijo(a)

adulto(a) siente

demasiado estrés por

cuidar a sus padres

de mayor edad, está

bien calmarlos con

medicación.

$100 \quad 17 \% \quad 64 \%$

$16 \%$

$3 \%$

Entre parejas de mayor edad, está bien golpearse o pegarse ocasionalmente.

Esta bien que un hijo(a)

adulto(a) que cuida a un padre o madre de mayor edad física o mentalmente incapacitado, le amarre a la cama $\quad 100$ 


\section{Abuso financiero}

Cuando un hijo(a)

adulto(a) pide dinero

prestado a sus padres

DE mayor edad, está

bien que no lo devuelvan

aún cuando los

padres se lo pidan. $\quad 100$

Está bien que un

hijo(a) adulto(a)

utilice el dinero de

sus padres para su

uso propio.

100

$7 \%$

$83 \%$

$10 \%$

$0 \%$

\section{Negligencia}

Esta bien que un

hijo(a) adulto(a) que

cuida a sus padres de

mayor edad encamados,

les deje solos

ocasionalmente por

unas horas.

$8 \%$

$74 \%$

$14 \%$

$0 \%$

Cuando los padres de mayor edad deciden no cuidarse a sí mismo y su salud empeora, los hijos(as) deben hacer algo.

$100 \quad 97 \% \quad 1 \%$

$2 \%$

$0 \%$

\section{Dilemas éticos de auto-determinación}

Cuando los padres

o madres de mayor

edad rechazan comer

continuamente está bien

que sus hijos adultos

les fuerce a comer. $\quad 100$ 
Aseveración

\section{N De Desacuerdo Acuerdo}

\section{Percepción sobre la Magnitud}

$\mathrm{El}$ abuso de

personas de mayor

edad es tan frecuente

como el abuso de

los niño(as).

$100 \quad 78 \%$

$13 \%$

$2 \% \quad 7 \%$

El abuso sexual en

las personas de mayor edad es tan frecuente como el abuso sexual en los niño(as)

$39 \%$

$45 \%$

$2 \%$

$14 \%$

7. Percepción sobre causas $\mathrm{El}$ abuso en personas de mayor edad es cometido principalmente por personas mentalmente enfermas o por drogadictos.

$49 \%$

$36 \%$

$7 \%$

$8 \%$

Muchas personas de mayor edad son maltratadas porque hicieron algo mal y lo merecen.

$79 \%$

$3 \%$

$6 \%$

$\mathrm{El}$ abuso de personas

de mayor edad es ocasionado

principalmente

por miembros

de su familia.

$69 \%$

$17 \%$

$9 \%$

$5 \%$

Los padres (madres) de mayor edad que abusaron a sus hijos de pequeños, merecen el abuso de sus hijo(as) cuando crecen. 


\section{Actitudes hacia reportar el abuso}

Cuando un miembro

de la familia comete

abuso o negligencia

hacia adultos de

mayor edad, las

personas fuera de

la familia no

$\begin{array}{llllll}\text { deben meterse. } & 100 & 22 \% & 71 \% & 5 \% & 2 \%\end{array}$

Cuando un vecino

sabe que un adulto de

mayor edad es abusado

por un miembro

de su familia, el vecino

no debe informarlo

a las autoridades. $\quad 100$

100

$6 \% \quad 88 \%$

$5 \%$

$1 \%$

Cuando un vecino

sospecha que un

adulto de mayor

edad es abusado

por un miembro

de su familia,

el vecino no debe

reportarlo a las

autoridades hasta

no estar seguro.

100

$57 \%$

$39 \%$

$3 \%$

$1 \%$

Reportar a los

que abusan de

personas de

mayor edad a las

autoridades, destruiría

la vida de los

maltratadores.

$3 \%$

$4 \%$ 


\section{B: Cubanos:}

Aseveración

\section{Abuso Psicológico}

Esta bien que un

hijo adulto le grite

ocasionalmente a

sus padres o madres

ancianas.
N

De D

Desacuerdo

Depende

No Sé Acuerdo

Ser ignorado, excluido o aislado por

un miembro de la

familia es tan dañino

como ser abusado

físicamente.

100

$0 \%$

$100 \%$

$0 \%$

$0 \%$

2. Abuso Físico

Cuando un hijo(a)

adulto(a) siente

demasiado estrés

por cuidar a sus padres

de mayor edad,

está bien calmarlos

con medicación.

$73 \%$

$15 \%$

$0 \%$

Entre parejas de mayor

edad, está bien

golpearse o pegarse

ocasionalmente.

$99 \%$

$1 \%$

$0 \%$

Esta bien que un

hijo(a) adulto(a)

que cuida a un padre

o madre de mayor edad

física o mentalmente

incapacitado, le

amarre a la cama.

$8 \%$

$77 \%$

$12 \%$

$3 \%$ 


\section{Abuso financiero}

Cuando un hijo(a)

adulto(a) pide dinero

prestado a sus padres

de mayor edad, está

bien que no lo

devuelvan aún cuando

los padres se lo pidan. 100

$25 \% \quad 66 \%$

$3 \%$

$6 \%$

Está bien que un

hijo(a) adulto(a)

utilice el dinero

de sus padres para

su uso propio.

100

$9 \% \quad 80 \%$

$11 \%$

$0 \%$

\section{Negligencia}

Esta bien que un

hijo(a) adulto(a) que

cuida a sus padres de

mayor edad encamados,

les deje solos

ocasionalmente

por unas horas.

$6 \%$

$73 \%$

$21 \%$

$0 \%$

Cuando los padres

de mayor edad deciden

no cuidarse a sí mismo

y su salud empeora,

los hijos(as) deben

hacer algo.

$97 \%$

$0 \%$

$0 \%$

$3 \%$

5. Dilemas éticos de auto-determinación

Cuando los padres o madres de mayor edad

rechazan comer

continuamente está

bien que sus hijos adultos

les fuerce a comer. $\quad 100$

$12 \% \quad 73 \%$

$15 \%$

$0 \%$ 
Aseveración

\section{N D}

6. Percepción sobre la Magnitud

El abuso de personas

de mayor edad es tan

frecuente como el

abuso de los niño(as)

100

$33 \%$

$44 \%$

$2 \%$

$21 \%$

El abuso sexual en

las personas de mayor

edad es tan frecuente

como el abuso sexual

en los niño(as).

$100 \quad 23 \% \quad 66 \%$

$2 \%$

$9 \%$

\section{Percepción sobre causas}

El abuso en personas

de mayor edad

es cometido

principalmente

por personas

mentalmente

enfermas o por

drogadictos.

100

$39 \% \quad 30 \%$

$6 \%$

$25 \%$

Muchas personas de mayor edad son maltratadas porque hicieron algo mal y lo merecen.

El abuso de

personas de mayor edad es ocasionado principalmente por miembros

de su familia.

$39 \% \quad 33 \%$

$12 \%$

$15 \%$

Los padres (madres) de mayor edad que abusaron a sus hijos de pequeños, merecen el abuso de sus hijo(as) cuando crecen. 
Aseveración

\section{N De Desacuerdo Acuerdo}

Depende No Sé

8. Actitudes hacia reportar el abuso

Cuando un miembro

de la familia comete

abuso o negligencia

hacia adultos de mayor

edad, las personas

fuera de la familia no

deben meterse.

Cuando un vecino

sabe que un adulto de

mayor edad es abusado

por un miembro

de su familia, el

vecino no debe

informarlo

a las autoridades.

100

$30 \%$

$60 \%$

$7 \% \quad 3 \%$

Cuando un vecino

sospecha que un

adulto de mayor

edad es abusado

por un miembro

de su familia, el

vecino no debe

reportarlo a las

autoridades hasta

no estar seguro.

100

$73 \% \quad 12 \%$

$15 \%$

$0 \%$

Reportar a los que

abusan de personas

de mayor edad a las

autoridades, destruiría

la vida de los

maltratadores.

100

$35 \%$

$53 \%$

$6 \%$

$6 \%$ 


\section{Abuso psicológico}

Dos aseveraciones del cuestionario a las cuales se les pedía responder a las personas entrevistadas fueron diseñadas para lidiar con lo que típicamente se refiere al abuso psicológico. Una tenía que ver con los hijos gritarle a las personas ancianas y la otra con ser ignorado, excluido o aislado por miembros familiares. Respecto a estos dos asuntos, se encontró que sólo $1 \%$ de la muestra puertorriqueña y ninguno de los cubanos estuvo de acuerdo con que gritarle a las personas ancianas está bién. Respecto a ser ignorado, excluido o aislado, $92 \%$ de los puertorriqueños y un $85 \%$ de los cubanos estuvieron de acuerdo en que era tan dañino como ser abusado físicamente. Un $18 \%$ de ambas nuestras estuvo en desacuerdo.

\section{Abuso físico}

Tres aseveraciones se referían a lo que los analistas han determinado que es abuso físico hacia las personas ancianas. Una tiene que ver con que está bien que se medique a las personas ancianas para tranquilizarles, la otra con la aceptabilidad de que entre parejas se golpeen uno al otro y finalmente con el asunto de que los hijos amarren a los padres a la cama. Los entrevistados puertorriqueños y cubanos básicamente estuvieron generalmente en desacuerdo con que se medicara a las personas, (64\% y $73 \%$ respectivamente). Sin embargo, un $31 \%$ de ambos grupos respondió que dependía de la situación aunque no especificaron bajo que circunstancias era permitido, dejando la posibilidad abierta de que hubiesen sido testigos de situaciones similares o de que el uso de medicación es aceptable socialmente y no se ve como algo negativo.

Respecto a la idea de que el pegarse es aceptado entre parejas, sólo 2 personas en la muestra de puertorriqueños estuvo de acuerdo. Una persona entre los cubanos indicó que dependía. Un 90\% de los puertorriqueños estuvo en desacuerdo con que estaba bien amarrar a los padres física o mentalmente incapacitados a la cama y sólo un $3 \%$ estuvo de acuerdo. Por otro lado, un $73 \%$ de los cubanos estuvo en desacuerdo y $12 \%$ estuvo de acuerdo. Nuevamente entre ambas muestras tanto como un $21 \%$ indicó que si esto era o no aceptable dependía de situaciones en las que pueden mencionarse la seguridad de la persona anciana. 


\section{Abuso financiero}

Con respecto a una situación potencial de explotación financiera que envuelve a los hijos mayores, un porcentaje mayor o cercano al 25\% no consideraron esto como problemático. En específico, un $25 \%$ de la muestra cubana y $11 \%$ de los puertorriqueños estuvieron de acuerdo en que cuando los hijos le toman dinero prestado está bien que no se los devuelvan aún cuando los padres lo reclamen. Mientras un $66 \%$ de los cubanos y $60 \%$ de los puertorriqueño estuvieron en desacuerdo. El 27\% de la muestra de Puerto Rico y sólo un 3\% de los cubanos indicó que dependía.

Ambos grupos (80\% cubanos y $83 \%$ de PR) estuvieron primordialmente en desacuerdo en que los hijos usaran el dinero de sus padres para uso personal. Sólo un $7 \%$ de PR y $9 \%$ de Cuba estuvieron de acuerdo, y en este respecto debe tomarse en cuenta el sentir cultural de que "lo que es de los padres pertenece a los hijos", de parte de algunas personas de mayor edad en ambos países.. Nuevamente algunos señalaron que dependía (!0\% PR y 11\% Cuba).

\section{Negligencia}

La sección que tenía que ver con negligencia incluía una aseveración respecto a los padres encamados ser dejados solos por sus hijos y otra respecto a si los hijos debían o no intervenir cuando los padres deciden no cuidarse a sí mismos. Respecto a la primera aseveración, la respuesta de ambos grupos fueron bastante similares. Un $74 \%$ de los de PR y un $73 \%$ de los cubanos estuvieron en desacuerdo de que se debía dejar solos a los padres encamados. Sólo un $8 \%$ de los puertorriqueños y un $6 \%$ de los cubanos estuvieron de acuerdo. Nuevamente debe llamarse la atención a la columna de "depende". En ambos grupos (14\% y $21 \%$ ) mencionaron que si esta conducta era aceptable o no debía ser juzgada por las circunstancias. Respecto a la otra aseveración, en total un $97 \%$ en ambos grupos estuvieron de acuerdo en que los hijos debían hacer algo cuando los padres se negaban a cuidarse. Esto sugiere que el respeto que los hijos mayores deban tener a la autodeterminación de los padres a no cuidarse debe ser considerado inaceptable o no deseada.

\section{Dilemas éticos de autodeterminación}

La situación en la cual los padres mayores rechazan alimentarse continuamente señala un dilema ético al cual se enfrentan los hijos. Estos pueden escoger entre forzarlos a comer, que puede ser considerado 
una forma de abuso físico o respetar la decisión de los padres de rechazar alimentarse, que puede ser considerado una manera de ser negligente. En este respecto, ambos grupos mostraron respuestas bastante similares. Un $16 \%$ de los puertorriqueños y un $12 \%$ de los cubanos estuvieron de acuerdo con la aseveración. En desacuerdo estuvieron un $73 \%$ de los cubanos y un $65 \%$ de los de PR. Mientras ambos grupos señalaron (15\% y $19 \%$ ) que dependía de las circunstancias. Esto sugiere que los hijos deben tomar en consideración cosas tales como la condición física y mental del padre o madre.

\section{Percepción sobre la magnitud o seriedad}

Esta sección sobre percepción de magnitud o seriedad del problema se abordó en aseveraciones que se relacionaban a si el abuso a ancianos ocurría tan frecuentemente como el de niños y si el abuso sexual en las personas de mayor edad era tan frecuente como el de menores. En respuesta a estas aseveraciones, hubo bastante diferencia entre ambos grupos. Mientras que un $78 \%$ de los puertorriqueños estuvo de acuerdo con que el abuso de personas mayores era tan frecuente como el de los niños, sólo un 33\% de los cubanos estuvieron de acuerdo. Un $44 \%$ de los cubanos estuvieron en descuerdo comparado con $13 \%$ de los puertorriqueños. Las respuestas a la segunda aseveración fueron bastante similares, de acuerdo estuvieron el $39 \%$ en PR y $33 \%$ en Cuba. En desacuerdo $66 \%$ de los cubanos y $45 \%$ de los de PR.

\section{Percepción sobre causas}

Relativamente la mayoría de los entrevistados de ambos países (49\% y $39 \%$ ) estuvieron de acuerdo en que el abuso y maltrato es cometido principalmente por personas mentalmente enfermas o usuarios de drogas, y el $69 \%$ de los de PR y $39 \%$ de Cuba están de acuerdo que es cometido principalmente por miembros de la familia. Un porcentaje considerable en ambos países está en desacuerdo en atribuirle la causa del abuso a la víctima anciana. Setenta y nueve por ciento de los puertorriqueños y $71 \%$ de Cuba indicaron estar en desacuerdo de que la personas son maltratadas porque hicieron algo mal y lo merecen. Solo un $11 \%$ de PR y $15 \%$ de Cuba estuvieron de acuerdo en que los padres que abusaron a sus hijos merecían ser abusados 


\section{Actitudes hacia reportar el abuso}

Las actitudes hacia el envolvimiento de una persona no parte de la familia en sospecha de maltrato o notificar el maltrato se midió por medio de tres aseveraciones. Primero, el 31\% de los cubanos en contraste con $22 \%$ de los puertorriqueños consideraban que cuando un miembro de la familia comete abuso o negligencia, las personas fuera de la familia no deben meterse, mientras que el $52 \%$ y $71 \%$ respectivamente estuvieron en desacuerdo. En la situación de que un vecino conoce que una persona anciana está siendo maltratada, un porcentaje mayor ( $60 \%$ Cuba y $88 \%$ PR) indicó que debe ser notificado a las autoridades. Es interesante observar que las personas entrevistadas endosaban menos el envolvimiento de extraños en asuntos familiares que en reportarlos a las autoridades. (31\% y $22 \%$ vs. $57 \%$ y $73 \%$ ). Finalmente sobre una tercera parte $(35 \%)$ de ambos grupos de entrevistados estuvieron de acuerdo en que reportar a los que abusan de personas de mayor edad a las autoridades destruiría la vida de éstos. Mientras, más de la mitad en ambos grupos estuvo en desacuerdo con esta aseveración.

\section{Conclusiones y recomendaciones}

El conocimiento sobre la percepción del abuso y maltrato puede servir de mucha ayuda a los profesionales en su intervención con personas ancianas víctimas de abuso y maltrato o violencia familiar. Las percepciones y experiencias de lo que las mismas personas ancianas entienden constituye el maltrato son importantes para desarrollar un contexto que permita entender no sólo los casos extremos de maltrato, sino también los marginales.

Los profesionales de servicios humanos deben estar alerta y tener conocimiento de intervenciones apropiadas que puedan aplicarse en situaciones donde se sospecha abuso a este sector poblacional. Las familias cuyos miembros mayores de edad requieren cuidado especial y continuo, tienen más probabilidades de experimentar tensión, particularmente si carecen de ayuda externa. La falta de ayuda o apoyo puede llevar al maltrato, abuso o negligencia en algunos ambientes familiares.

En la actualidad, las persons ancianas tanto puertorriqueñas como cubanas que necesitan cuidado prolongado, dependen grandemente de sus familias para asistencia más que otros adultos. No está muy claro el efecto que tiene en el cuidador o cuidadora la carga econó- 
mica, emocional o física que envuelve el cuido a estas personas. La tendencia de la población anciana de vivir junto a sus familiares (en sus casas o las de ellos) nos lleva a poner énfasis en modalidades de servicio que incorporen a la familia en el mantenimiento y tratamiento de la anciana, dando prioridad a facilitar la provisión de ayuda mediante asistencia en el hogar y servicios de ayuda temporeros. Las medidas preventivas, principalmente la educación son las mejores alternativas contra el abuso a personas de mayor edad. Debe también darse atención al fortalecimiento de los sistemas de apoyo comunitario que propicien una mejor calidad de vida.

\section{Referencias}

Anetzbeyer, G.T. (1987). The etiology of elder abuse by adult offspring. Springfield, Illinois: Charles C. Thomas.

Arroyo, N., E. Arroyo, N. Aybar, L. Carrión, F. Reyes, Y. Rodríguez y M. Torres (1992). El Maltrato a Envejecientes en Puerto Rico. Tesis de Maestría sin publicar, Escuela Graduada de Trabajo Social: Universidad de Puerto Rico.

Baba, J.; M. Colón; C, Cruz (1996). Violencia conyugal y la adultez tardía. Tesis de Maestría sin publicar, Escuela Graduada de Trabajo Social: Universidad de Puerto Rico.

Chang, J. y A. Moon (1997). Korean American elderly's knowledge and perceptions of elder abuse: A qualitative analysis of cultural factors. Journal of Multicultural Social Work. Vol. 6 (1/2), 139-154.

Griffin, L.W. (1994). Elder mistreatment among rural African-Americans. Jr. of Elder Abuse and Neglect. 6(1) 1-27.

Moon, A., y William, O.J (1993). Perceptions of elder abuse and helpseeking patterns among African-american, caucasian and Koreanamerican elderly. The Gerontologist. 33(3), 386-394.

Muñoz, M. (1985). El maltrato a ancianos en la familia. Tesis de Maestría sin publicar Facultad de Educación, San Juan:Universidad de Puerto Rico.

Oficina del Gobernador para Asuntos de la Vejez (1996). Informe Anual del Programa de Ombudsman de cuidado de larga duración. San Juan, Puerto Rico. 
Pillemer, K., y Finkelhor, D. (1988). The prevalence of elder abuse: A random sample survey. The Gerontologist. 28 (1), 51-57.

Quinn y Tomita, S.K. (1997). Elder abuse and neglect: Causes, diagnosis and intervention strategies $\left(2^{\text {nd }}\right.$. ed.) NY: Springer Publishing, Co.

Ramos-Tossas, H. (1991). Indicadores de Maltrato en una población de hombres y mujeres viejos en los centros de cuidado prolongado y cuidado diurno en la ciudad de Ponce. Tesis Doctoral sin publicar, San Juan:Centro de Estudios Caribeños.

U.S. Bureau of the Census (1991). Current population rports. March 1991, Serie p-20, No. 450, Washington, DC:US. Bureau of the Census. 